\title{
Grading of intensity of fluorescence in anti-nuclear antibody test
}

\author{
Therese Mary Dhason ${ }^{1, *}$, Meenakshi Subramaniam², Sowndhariya Velu Annamalai ${ }^{3}$, Vignesh Mantharam ${ }^{4}$, \\ Nesa Aurlene Jayadhas ${ }^{5}$
}

${ }^{1}$ Associate Professor, Dept. of Microbiology, Government Dharmapuri Medical College, The Tamil Nadu Dr. MGR Medical University, Chennai, Tamil Nadu, ${ }^{2}$ Associate Professor, ${ }^{3,4}$ Post Graduate, ${ }^{2}$ Dept. of Microbiology, ${ }^{3,4}$ Dept. of Rheumatology, Madras Medical College, The Tamil Nadu Dr. MGR Medical University, Chennai, Tamil Nadu, ${ }^{5}$ Post Graduate, Dept. of Public Health Dentistry, SRM Dental College, SRM University, Chennai, Tamil Nadu, India

*Corresponding Author: Therese Mary Dhason

Email: tmary1964@gmail.com

Received: $29^{\text {th }}$ September, 2018

Accepted: $26^{\text {th }}$ November, 2018

\begin{abstract}
Introduction: The gold standard method to detect anti-nuclear antibodies is Indirect Immunofluorescence. Hep-2 cell lines coated slides are used to identify and detect the various patterns of fluorescence produced by anti-nuclear antibodies. Interpretation is based on the titre, pattern and intensity of fluorescence.

Objectives: To find out whether any relationship exists between the intensity of fluorescence and the concentration of anti-nuclear antibodies. For this the samples which exhibited homogeneous pattern were further tested by quantitative Enzyme linked immunosorbent assay to find out the concentration of anti-dsDNA antibody.

Materials and Methods: This prospective study was carried over a period of one year from January 2017 to December 2017 and the study group included 500 patients with lupus and 100 age and sex matched healthy controls. All the samples were processed for Indirect Immunofluorescence. The samples which were of homogeneous pattern were tested by Enzyme linked immunosorbent assay to quantitate the anti-dsDNA antibody concentration.

Results: The anti-dsDNA positivity in homogeneous pattern was statistically significant $(\mathrm{p}<0.00001)$ when compared with other patterns. The association between intensity of fluorescence and mean antibody concentration was statistically not significant ( $\mathrm{p}$ 0.5918).

Conclusion: As the intensity of fluorescence dose not reflect the concentration of antibody, further studies are needed to elucidate whether grading of intensity is mandatory in interpretation.
\end{abstract}

Keywords: Anti-nuclear antibodies, Indirect Immunofluorescence, Intensity grading, Antibody concentration.

\section{Introduction}

Autoimmune Diseases: Auto immune diseases are either organ specific or systemic and involve a number of organs and tissues. In auto immune disorders immune dysregulation occurs leading to hyperactive $\mathrm{T}$ and $\mathrm{B}$ cells. Affected individuals produce autoantibodies to a vast array of tissue antigens.

Autoantibody Detection Methods: Autoantibodies in various autoimmune disorders are detected by the immunoassays like Enzyme linked immunosorbent assay (ELISA), Indirect Immuno fluorescence (IIF), Radio Immuno Assay (RIA)and Line Immuno assay (LIA). For detecting the antinuclear antibodies and anticytoplasmic antibodies in autoimmune rheumatic diseases, IIF is the gold standard screening method. These antibodies serve as disease markers though the role of these autoantibodies in the pathogenesis of the disease remains controversial. ${ }^{1}$ Autoantibodies in Rheumatic diseases comprise of anti- dsDNA, anti histone, anti Sm and a lot more.

Autoantibodies in Lupus: In Systemic Lupus Erythematosus, the lupus specific autoantibodies like anti-dsDNA, anti Smith antibodies, anti histone antibodies and the other antibodies present can be detected by Indirect Immunofluorescence. Even though anti-dsDNA antibodies have a significant correlation with SLE, other antibodies to nuclear antigens aid in the diagnosis and assessing the prognosis of various connective tissue disorders like Progressive Systemic Sclerosis, Mixed Connective Tissue Disease (MCTD), Sjogren's Syndrome and Polymyositis. ${ }^{2}$ Thus, the Antinuclear antibody (ANA) diagnostic test is helpful not only for SLE, but also in screening for other connective tissue disorders. ${ }^{3}$

Antigen Substrates in ANA-IIF: Earlier the substrates used for testing ANA by IIF were mouse liver and primate liver. Since the 1970s Hep2 cells derived from epithelial cells of laryngeal carcinoma are being increasingly used in most of the laboratories. Now the standard substrate for ANA by Indirect Immuno fluorescence is Human epithelial cells. The different substrates available are Hep2, Hep2000 and Hep2010.

ANA Results Reporting Guidelines: To improve the standards of the reporting system the Autoantibody Standardization Committee has collaborated with the Centers for Disease Control and Prevention (CDC) and other agencies to formulate autoantibody reference standards. Till date, there are 17 reference standards which are accessible to laboratory personnel free of cost. This is to improve the laboratory quality control of ANA and other autoantibody testing. ${ }^{4}$ However, the problems in ANA testing and reporting still prevails. While 
reporting, the titer followed by the intensity of fluorescence and the pattern of fluorescence observed are mentioned. In places where testing with the serial dilutions of the samples is not feasible, the test is done in a single dilution which is significant as per the guidelines of the kit. Grading of the intensity of fluorescence is performed based on the guidelines established by the Centers for Disease Control (CDC), Atlanta, Georgia. ${ }^{5}$ The maximum intensity with brilliant yellow green fluorescence is graded as $4+$. less brilliant yellow green fluorescence as $3+$. definite but dull yellow green fluorescence is $2+$ and very dim subdued fluorescence is $1+$. With regards to the pattern of nuclear or cytoplasmic fluorescent staining, an intensity of $1+$ or greater with a clearly discernible pattern is reported as positive.

Nuclear patterns are defined as any staining of the Hep-2 interphase cell nuclei irrespective of positive or negative staining of mitotic cells. Depending upon the staining of the nuclear components there are 6 major pattern groups and 11 minor pattern subgroups. Staining of the cytoplasm of Hep-2 cells irrespective of the mitotic staining is interpreted as cytoplasmic pattern. ${ }^{6} \mathrm{~A}$ positive and negative antibody control must be included with each run to validate the test results, The cells should exhibit less than $1+$ fluorescence in negative control and fluorescence intensity of $3+$ or $4+$ with a discernible pattern in positive control. Diffuse staining of the nucleus with or without nucleolar staining and bright positive staining of chromosome region of mitotic cells is known as Homogeneous pattern. The antibodies which are produced against ds-DNA, nucleosomes or histones exhibit a homogeneous pattern. ${ }^{7}$

In our Centre samples are tested in 1/80 dilution. The aim of the study was to find out the relevance of grading the intensity of fluorescence. The samples which had a homogeneous pattern in IIF were tested for antidsDNA antibody concentration by ELISA test and the intensity of fluorescence was compared with the antibody level.

\section{Materials and Methods}

Materials: A prospective study was done at Rajiv Gandhi Government General Hospital for a period of 1 year from January 2017 to December 2017. Study population included both sexes of age group 10-50 years. Methods: A total of 600 blood samples, 500 from diseased group and 100 from age and sex matched healthy individuals were collected. Blood samples were processed and tested for antinuclear antibody by Indirect Immuno fluorescence using Hep 2000 substrate procured from Immuno concepts (Sacramento, CA). The 240 samples which showed homogeneous pattern in the diseased group were processed for anti-ds DNA ELISA. ELISA test was done using $\mathrm{NcX}$ anti-dsDNA ELISA kits manufactured by EURO IMMUN (Germany). Samples which were anti- ds DNA positive were analyzed to find out the relationship between the intensity of fluorescence and the antibody concentration. In the same way the 100 samples from healthy controls were processed.

\section{Statistical Analysis}

Statistical analysis was done using SPSS. ChiSquare test of association was used to test the association between homogeneous pattern and other patterns with anti-dsDNA positive and anti- dsDNA negative patients. Pearson's correlation test was applied to test for the correlation between intensity of fluorescence and mean antibody concentration.

\section{Results}

Antibody to any one of the nuclear antigens is known as antinuclear antibody. Most of the autoimmune diseases especially systemic lupus erythematosus present with antinuclear antibodies in serum. When these antibodies are present, a definite pattern with apple green fluorescence is observed in Indirect Immunofluorescence. The pattern of fluorescence and intensity of fluorescence in 1/80 dilution were studied and the ANA positivity among the study group is given in Fig. 1.

Though different patterns of fluorescence like coarse speckled, homogeneous, SSA, nucleolar and centromere were observed, samples showing homogeneous pattern were further processed to detect the anti-dsDNA antibody concentration. The number of diseased showing homogenous pattern in IIF was compared with healthy controls showing homogenous pattern. (This is given in Fig. 2)

The initial screen for ANA by IIF is to determine the need for further antibody specificity testing or to distinguish false positive ELISA. In our study all the samples showing homogenous pattern were processed for anti-dsDNA ELISA. A quantitative anti-dsDNA ELISA test was performed. The anti-dsDNA positivity in homogenous and other patterns in the diseased group is given in Table 1 .

The purpose of our study was to find out the relationship between fluorescence intensity grading and antibody concentration. Homogeneous pattern with $4+$, $3+2+$ and $1+$ intensity was segregated and their mean anti-dsDNA antibody concentration was correlated with the intensity of fluorescence (This is denoted in Table 2).

Table 1: Anti-dsDNA positivity in homogenous and other patterns:

\begin{tabular}{|l|c|c|}
\hline Patterns & $\begin{array}{c}\text { anti-dsDNA } \\
\text { Positive }\end{array}$ & $\begin{array}{c}\text { anti-dsDNA } \\
\text { negative }\end{array}$ \\
\hline $\begin{array}{l}\text { Homogenous } \\
\text { patterns (n240) }\end{array}$ & 200 & 40 \\
\hline $\begin{array}{l}\text { Other } \\
\text { patterns(n180) }\end{array}$ & 21 & 159 \\
\hline
\end{tabular}

Chi value $=211.89$, p-value $-<0.00001^{* *}$ 
Table 2: Intensity of fluorescence versus ant-dsDNA concentration (n 200)

\begin{tabular}{|c|c|}
\hline $\begin{array}{c}\text { Intensity of } \\
\text { fluorescence }\end{array}$ & $\begin{array}{c}\text { Mean Antibody } \\
\text { Concentration }\end{array}$ \\
\hline 1 & 526 \\
\hline 2 & 538 \\
\hline 3 & 540 \\
\hline 4 & 532 \\
\hline
\end{tabular}

$\mathrm{r}=0.4082, \mathrm{p}$-value -0.5918

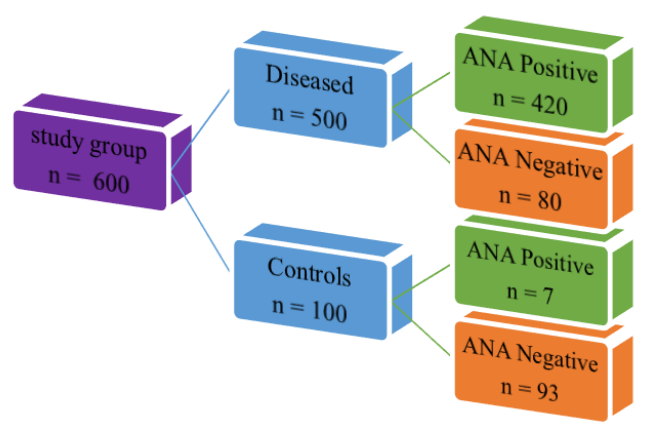

Fig. 1: ANA positivity in study group

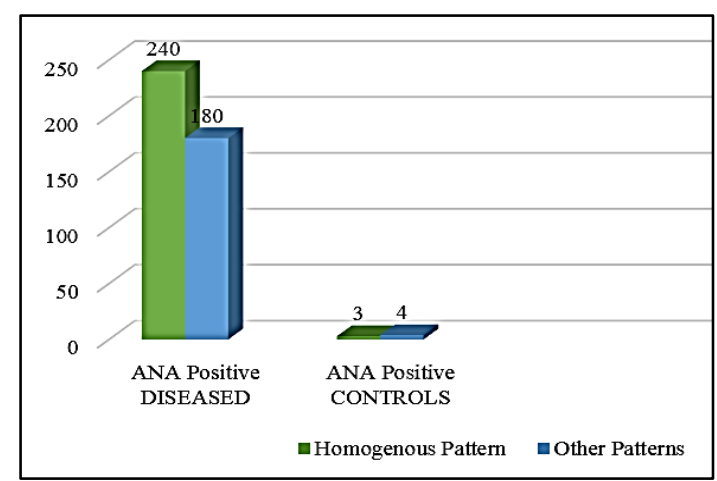

Fig. 2: Patterns of fluorescence in indirect immunofluorescence

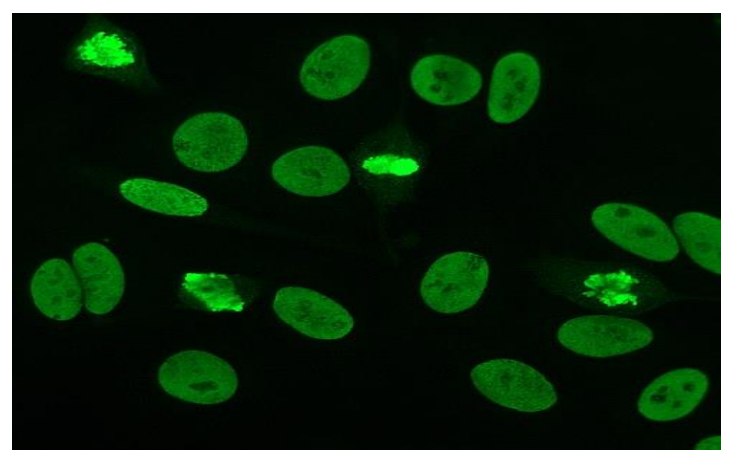

Fig. 3: Homogeneous pattern

\section{Discussion}

Antinuclear Antibodies: Antinuclear antibodies (ANA) are often detected in autoimmune rheumatic or connective tissue disorder and it is positive in most of the SLE patients. Low titers of ANA are found in infectious diseases, severe burns and in healthy individuals especially the elderly personnel.
As the ANA screen is a nonspecific test, all positive samples should be processed further to end point dilution to distinguish low titer ANA. These samples also should be tested for the specific antibodies like anti-dsDNA and Extractable nuclear antibodies (ENA) either by ELISA or other method. ${ }^{8}$

The diagnostic tests adopted in UK to detect antidsDNA antibody are ELISA, Crithidia luciliae IIF or Radio immuno assay. These specific tests aid in the diagnosis and the assays with high sensitivity are done to monitor disease activity. ${ }^{9}$

Various substrates are being used in ANA indirect immunofluorescence. Substrates like Hep2 cells, Hep 2000, Hep 2010 are available. In Hep 2 cells, the wells are coated with human epithelial cells cultured from laryngeal carcinoma. The expression of Sjogren's syndrome A (SSA) antigen in Hep 2 cells is less. To enhance the SSA expression Hep 2 cells transfected with SSA antigen named as Hep 2000 is being used. A biochip which carries hep 2 cells and frozen sections of primate liver manufactured by EURO IMMUN (Germany) is called as Hep 2010. The advantage of Hep 2010 are its ability to pick up true negatives and the nuclear membrane pattern produced by anti lamin antibodies. Our Laboratory uses Hep 2000 substrate, so that antibody to SSA is not missed out. The sensitivity of Human epithelial cell substrate cells in anti nuclear antibody detection is much more than tissue sections and pattern recognition are good on Human epithelial cells. ${ }^{10}$ Based on the staining patterns they are classified into nuclear, cytoplasmic and mitotic patterns. Some of the patterns are recognized for mandatory reporting while others are for expert-level reporting as per the Brazilian Consensus Strategy. ${ }^{11}$

ICAP Guidelines: According to ICAP, the patterns which are recognized easily are competent level and the patterns which are challenging to the reporting personnel are expert level. The six competent level nuclear patterns include homogeneous, speckled, dense fine speckled (DFS), centromere, nuclear dots and nucleolar. The cytoplasmic patterns which are competent- level reportable are fibrillar, speckled, reticular/ mitochondrion like, polar / Golgi like and rods and rings (RR). The RR patterns are detected consistently in slides manufactured from only some manufacturers. ${ }^{12,13}$

ANA Titers: To ensure the potency of the reagents and for legitimate reporting a positive control and a negative control is run along with test samples. Subjective errors are possible in ANA interpretation unless the person reporting is skilled and knowledgeable about the patterns, grading and titer. A complete report includes the titer, intensity of fluorescence and the pattern. As per the MBL BION Anti-nuclear antibody test system 1/40 and $1 / 80$ are low titers; $1 / 160$ and $1 / 320$ are medium titers and1/640 and above are high titers. Each laboratory should establish its own ranges. The standard dilution adopted in our laboratory is $1 / 80$ as specified in the kit protocol. When a definite pattern is observed in this titer 
we report it as positive. The patterns which are recognized very often in our center are homogeneous, speckled, nucleolar, centromere and cytoplasmic speckled. The Golgi like pattern was observed in 2 samples and centriole the mitosis specific antibody pattern (MSA) was picked up in 2 samples. Intensity was graded as per CDC guidelines.

In our study, antinuclear antibody was positive in $84 \%$ of the diseased group. Patients on steroid therapy or in remission may present with a negative ANA ${ }^{[3]}$. Hence ANA negative in the rest of the diseased population can be attributed to either of these. Homogeneous pattern of fluorescence was observed in 57\% of the ANA positive samples. The antibodies which produce homogeneous pattern are anti-dsDNA, anti-histone and antinucleosome antibodies. To detect the presence of antidsDNA antibodies in samples with homogeneous pattern, monospecific anti-dsDNA ELISA test was done by quantitative method. Out of the 240 samples, antidsDNA was positive in 200 samples. Hence the rest of the samples can be either positive for anti-histone or antinucleosome antibodies. As the institutions has resources constraint, these tests were not performed.

The primary aim of our study was to find out the necessity of grading the intensity of fluorescence while reporting. The present study focused on the relationship between the concentration of anti-dsDNA antibody and the intensity of fluorescence. The mean anti-dsDNA antibody concentration of samples with $1+, 2+, 3+$ and $4+$ intensities of fluorescence was calculated and statistical analysis was done to find out the significance of fluorescence intensity grading. From the statistical analysis it was found that the mean antibody concentration in various grades of fluorescence intensity was almost the same and it was noted that intensity was not relevant in clinical perspectives. Differences in fluorescence microscope optics, filters and light sources may result in differences in the intensity when observing the same slide using different microscopes. ${ }^{14}$ It is also subjective when grading the intensity. From our experience, the LED epifluorescence microscopes were found to be far superior than the mercury vapor lamp microscopes. Pattern recognition is excellent and the intensity of fluorescence is good with LED microscopes.

\section{Conclusion}

Antinuclear antibody by IIF remains to be the gold standard screening method to detect ANA. All homogeneous patterns should be further tested by specific assays to confirm the presence of anti- dsDNA antibody. As the intensity of fluorescence was not directly proportional to the concentration of antibodies in our study, further studies are required in this to elucidate the significance of including fluorescence intensity grading system in the standard reporting format.

\section{Conflict of Interest: None.}

\section{References}

1. Tan EM. Antinuclear antibodies: diagnostic markers for autoimmune diseases and probes for cell biology. Advances in immunology. 1989;44:93-151.

2. Wolfe JF, Adelstein E, Sharp GC. Antinuclear antibody with distinct specificity for polymyositis. Journal of Clinical Investigation. 1977;59(1):176

3. Nakamura RM, Peebles CL, Molden DP, Tan EM. Advances in laboratory tests for autoantibodies to nuclear antigens in systemic rheumatic diseases. Laboratory Medicine. 1984;15(3):190-8.

4. Chan EK, Fritzler MJ, Wiik A, Andrade LE, Reeves WH, Tincani A, Meroni PL. AutoAbSC. Orgautoantibody standardization committee in 2006. Autoimmunity reviews. 2007;6(8):577-80.

5. Lyerla HC, Forrester FT. Immunofluorescence methods in virology. Dept. of Health, Education, and Welfare, Public Health Service, Center for Disease Control, Bureau of Laboratories, Laboratory Training and Consultation Division, Virology Training Branch. 1979:71-81.

6. Report of the first international consensus on standardized nomenclature of antinuclear antibody Hep-2 cell patterns 2014-2015 Aug 20:4.

7. Report of the first international consensus on standardized nomenclature of antinuclear antibody Hep-2 cell patterns 2014-2015 Aug 20:7.

8. Casals SP, Friou GJ, Myers LL. Significance of antibody to DNA in systemic lupus erythematosus. Arthritis \& Rheumatology. 1964;7(4):379-90.

9. Emlen W, O'neill L. Clinical significance of antinuclear antibodies. Comparison of detection with immunofluorescence and enzyme-linked immunosorbent assays. Arthritis \& Rheumatology. 1997;40(9):1612-8.

10. McCany GA, Rice JR. Charecterization and comparison of commercially available antinuclear antibody kits using single pattern index sera. J Rheum. 1980;(7):339-347.

11. Francescantonio PL, de Melo Cruvinel W, Dellavance A, Andrade LE, HurTaliberti B, Von Mühlen CA, Bichara CD, Bueno C, Mangueira CL, Carvalho DG, Eloísa SD. IV Brazilian guidelines for autoantibodies on HEp-2 cells. Revista Brasileira de Reumatologia. 2014;54(1):4450.

12. Covini G, Carcamo WC, Bredi E, von Muhlen CA, Colombo M, Chan EK. Cytoplasmic rods and rings autoantibodies developed during pegylated interferon and ribavirin therapy in patients with chronic hepatitis $\mathrm{C}$. Antivir Ther. 2012;17(5):805-11.

13. Keppeke GD, Nunes E, Ferraz ML, Silva EA, Granato C, Chan EK, Andrade LE. Longitudinal study of a human drug-induced model of autoantibody to cytoplasmic rods/rings following HCV therapy with ribavirin and interferon- $\alpha$. PloS one. 2012;7(9): e45392.

14. Cleymaet, JE, Nakamura RM. Indirect immunofluorescent antinuclear antibody tests: Comparison of sensitivity and specificity of different substrates. American Journal of Clinical Pathology. 1972;58(4):388-93.

How to cite this article: Dhason T. M, Subramaniam
M, Annamalai S V, Mantharam V, Jayadhas N A.
Grading of intensity of fluorescence in anti-nuclear
antibody test. Indian J Microbiol Res. 2018;5(4):512-
515 .

